\title{
Returning to the emergency room: An analysis of emergency encounters following urologic outpatient surgery
}

Luke Witherspoon, MD, MSc${ }^{1}$; Rodney H. Breau, MD, MSc ${ }^{1,2}$; Christopher Langley, MD ${ }^{1}$;

Ranjeeta Mallick, $\mathrm{PhD}^{2}$; Ilias Cagiannos, $\mathrm{MD}^{1}$; Christopher Morash, $\mathrm{MD}^{1}$; J. Stuart Oake, $\mathrm{MD}^{1}$; Luke T. Lavallée, MDCM, MSc ${ }^{1,2}$

${ }^{1}$ Division of Urology, Department of Surgery, The Ottawa Hospital and University of Ottawa, Ottawa, ON, Canada; ${ }^{2}$ Ottawa Hospital Research Institute, Ottawa, ON, Canada

Cite as: Witherspoon L, Breau RH, Langley C, et al. Returning to the emergency room: An analysis of emergency encounters following urologic outpatient surgery. Can Urol Assoc J 2021 March 18; Epub ahead of print. http://dx.doi.org/10.5489/cuaj.7063

Published online March 18, 2021

$* * *$

\section{Abstract:}

Introduction: Previous reports indicate urologic surgeries are associated with high rates of hospital re-admission. This study aims to identify factors associated with emergency room (ER) encounters following urologic outpatient surgery.

Methods: All outpatient surgeries performed at The Ottawa Hospital between April 1, 2008, and March 31, 2018 by urology, general surgery, gynecology, and thoracic surgery were identified. All ER encounters within 90 days of surgery were captured. Rates of ER encounters by surgical service and procedure type were determined. Patient and surgical factors associated with ER encounters were identified. Factors included age, sex, marital status, presence of primary care provider, procedure, and American Society of Anesthesiologists (ASA) score.

Results: A total of 38377 outpatient surgeries by the included surgical services were performed during the study period, of which urology performed 16552 (43.1\%). Overall, 5641 (14.7\%) ER encounters were identified within 90 days of surgery, including 2681 (47.5\%) after urologic surgery. On multivariable analysis, higher ASA score IV vs. I was associated with higher risk of ER encounter (relative risk [RR] 1.95, 95\% confidence interval (CI) 1.46-2.5) and being married was associated with a lower risk of ER encounter (RR 0.85, 95\% CI 0.77-0.93). Urologic surgeries with the highest risk of ER encounters, compared to the lowest risk procedure (circumcision), were greenlight laser photo vaporization of the prostate (PVP) (RR 3.2, 95\% CI 
1.8-5.61), ureteroscopy (RR 3.2, 95\% CI 1.9-5.4), and ureteric stent insertion (RR 3.1, 95\% CI 1.8-5.5).

Conclusions: ER encounters following outpatient surgery are common. This study identifies risk factors to recognize patients that may benefit from additional support to reduce ER care needs.

\section{Introduction}

Readmission to hospital following outpatient surgery is closely monitored by individual hospitals and is a commonly used metric when assessing quality of care. In the United States, it is estimated that post-surgical readmissions cost Medicare more than 17 billion US dollars per year $^{1}$, and hospitals are now being sanctioned for excess readmissions. ${ }^{2}$

Post-operative patients presenting to the emergency room (ER) but who are not readmitted, also use significant health care resources, and have not been well characterized. Most reported studies have focused on short post-discharge periods (24-72 hours), or on patientreported data. ${ }^{3,4}$ Of the studies assessing post-operative patients returning to emergency rooms, urological patients are noted to have high ER encounter rates. ${ }^{5}$

Health care organizations are interested in transitioning to more outpatient surgeries for economic and logistical reasons, with an increasing trend in outpatient surgeries worldwide. ${ }^{6,7}$ In doing so, it is imperative to determine that patient safety, resource usage and cost are not negatively impacted by such changes. While previous studies have identified urology as a significant contributor to ER encounters and admissions after surgery, little has been reported about why these patients are returning. ${ }^{5}$ Currently available provincial databases that track readmission data have focused on more invasive oncologic procedures, leaving clinicians with a limited understanding of the outcomes after outpatient surgeries. ${ }^{8}$

By definition outpatient surgeries are completed on patients that the medical profession has deemed fit to undergo surgery and safety return home the same day. If these patients subsequently visit the emergency room to receive care frequently, perhaps the design of outpatient surgery programs are flawed, with either better patient selection or after-surgery supports needing improvement. With the paucity of data surrounding this patient population this study set out to determine the rate that urology patients return to the emergency room within 90 days of outpatient surgery, and to identify patient and surgical risk factors that may be targeted to improve care. With this information future study design can occur to further assess at risk populations, and aid in outpatient surgery care. 


\section{Methods}

\section{Study cohort and setting}

This is a single-center retrospective study. All patients undergoing outpatient urology surgery in the main operating room with anesthesia at The Ottawa Hospital (Ottawa, Canada) between April 1, 2008, and March 31, 2018, were included. All emergency surgeries and patients admitted to hospital following surgery were excluded. Outpatient surgery could include open, laparoscopic, and transurethral procedures. No restriction was placed on the type of procedure. All surgical procedures performed by general surgery, gynecology, and thoracic surgery were also captured in the same fashion for comparison. These services were selected as they perform surgeries in the abdomen and pelvis like urology, with a variety of surgical methods (endoscopic, laparoscopic, open). The Ottawa Hospital comprises three main hospital campuses capable of conducting outpatient surgery, and services a patient population of approximately 1.2 million annually.

The Ottawa Hospital maintains an integrated administrative data warehouse which is automatically populated for every patient interaction. This database was used to perform a retrospective analysis of patients who underwent outpatient surgery with the selected services.

\section{Statistical analyses}

Patient, surgical, and ER encounter characteristics were recorded. Patient characteristics included: age, sex, marital status, American Society of Anesthesiologists (ASA) score (score from 1-5 where a higher score indicates worse comorbidities), and documented presence of a primary care provider. Surgical characteristics included: procedure performed, and date of surgery. The primary outcome was the first occurrence of an ER encounter within 90 days from a patient's procedure, the recorded encounter descriptive characteristics included: number of ER encounters within 90 days, time from procedure to ER encounter in days, readmission to hospital within 24 hours of ER encounter, and reason for ER encounter or admission. A 90-day time period was used because some complications of surgery (ex: venous thromboembolism) may present in a delayed fashion. A sensitivity analysis was also performed using a 30-day cutoff to verify if rates changed meaningfully. Procedure type was classified based on the operative record at the end of the procedure by surgical teams. The top 10 most commonly performed urology procedures were identified based on case rates for further analysis as these represent the highest burden to the system and most opportunity for intervention (Supplemental Table 1). The reason for return to the ER or admission were abstracted from the data warehouse and were based on the patient's diagnosis at time of admission to hospital or discharge from the ER.

When appropriate mean (standard deviation) and median (lower quartile, upper quartile) were reported. Multivariable analyses were performed to determine associations (relative risk with 
95\% confidence interval) between patient and surgical characteristics and the first ER encounter within 90 days of surgery using a log binomial regression.

Institutional ethics board approval was obtained for this study. SAS software version 9.4 for Windows was used for the analyses (Cary, NC, USA).

\section{Results}

During the study period, 38,377 patients underwent outpatient surgery with anesthesia by the selected services at The Ottawa Hospital. Urology performed 16,552 (43.1\%) compared to general surgery 18,676 (48.7\%), gynecology 1,283 (3.3\%), and thoracic surgery 1,866 (4.9\%). Baseline patient characteristics are summarized in Table 1. Mean age for each service was: urology 63.3 years standard deviation (SD) (16.4), general surgery 52.9 (SD 15.48), gynecology 49.8 (SD 14.2), and thoracic surgery 64.6 (SD 12.4).

\section{Emergency encounters across surgical services}

During the study period, there were 5641 different ER encounters within 90 days of surgery with some outpatient surgeries being associated with more than one ER encounter. Urologic surgeries were associated with 2681 ER encounters within 90 days of the surgery. $16 \%$ of urologic surgeries subsequently had an ER encounter, with urologic surgery patients representing $47.5 \%$ of all ER encounters across all surgical specialties in the study cohort. General surgery had 2404 encounters (12.9\% of general surgery surgeries resulted in an ER encounter, representing 42.6\% of all surgical specialties ER encounters), gynecology had 116 encounters $(9.0 \%$ of gynecology surgeries resulted in an ER encounter representing $2.1 \%$ of all surgical specialties ER encounters), thoracic surgery had 440 encounters (23.6\% of thoracic surgeries resulted in an ER encounter representing $7.8 \%$ of all surgical specialties ER encounters) (Figure 1). Patients with multiple ER encounters within 90 days of surgery were captured. Urology had 876 requiring $\geq 2$ ER encounters within 90 days (5.3\% of urologic surgeries), compared to general surgery with 654 patients (3.5\% of total general surgery surgeries), gynecology with 25 (1.9\% of total gynecology surgeries) and thoracic surgery with 144 (7.7\% of total thoracic surgery surgeries). Most ER encounters occurred within 14 days of surgery including $51.4 \%$ of urology encounters with the ER (Table 1).

\section{Urologic surgeries associated $\mathrm{ER}$ encounters}

During the study period, peritoneal dialysis (PD) catheter insertion (158 encounters, 25\% rate of ER encounter post-surgery), TURBT (611 encounters, 17\% rate of ER encounter post-surgery) and ureteroscopy (549 encounters, 21\% rate of ER encounter post-surgery) were associated with the highest volumes and rates of return to the ER after urologic surgery (Figure 2). The median (lower quartile (LQ), upper quartile (UQ)) time to ER encounter after urologic surgery was 13 days (LQ 3, UQ 44). The five most common reasons for return to ER after urologic surgery as documented in the medical record were urinary retention (11\% of all encounters), hematuria 
( $10 \%$ of all encounters), disorder of urinary system ( $8 \%$ of all encounters), complication of procedure ( $7 \%$ of all encounters), and complications of a urologic device (5\% of all encounters). $176(6.6 \%)$ of all urology ER encounters post-surgery required admission to hospital, indicating that $93.4 \%$ did not require admission. The five most common reasons for readmission included bleeding event (17.6\% of all admissions), infective process (14.2\% of all admissions), acute pain ( $2.3 \%$ of all admissions), ureteric nephrolithiasis ( $2.3 \%$ of all admissions), and acute renal failure (1.7\% of all admissions).

A sensitivity analysis using a 30-day post-surgery cut-off for ER encounters identified a $10.8 \%$ rate of ER encounter (compared to $16 \%$ at 90 days). Similar to the recorded reasons for return to ER at 90 days, the five most common reasons for return to the ER after surgery within 30 days were retention of urine ( $14 \%$ of all encounters), hematuria ( $12.9 \%$ of all encounters), complication of procedure ( $8.9 \%$ of all encounters), disorder of urinary system ( $8.6 \%$ of all encounters), and complication of a urologic device (6.1\% of all encounters).

\section{Risk factors for return to the ER after urologic surgery}

Multivariable analysis revealed that a higher ASA score IV vs I (relative risk (RR), 95\% Confidence Interval (CI) RR 1.95 (95\% CI 1.46-2.5) was associated with higher rates of ER encounters. Patient sex, age, the year of surgery, and having a documented family physician were not associated with return to the ER (Figure 3). Being married was associated with a lower return to the ER after surgery RR 0.85 (95\% CI 0.77-0.93). Surgery type was associated with ER encounters. Compared to the procedure with the lowest risk of ER encounter which was circumcision (reference procedure), greenlight laser photo vaporization of the prostate (PVP) RR 3.2 (95\% CI 1.8-5.61), ureteroscopy RR 3.2 (95\% CI 1.9-5.4), ureteric stent insertion RR 3.1 (95\% CI 1.8-5.5), peritoneal dialysis (PD) catheter insertion RR 2.9 (95\% CI 1.7-5.1), and cystolithopaxy RR 2.82 (95\% CI 1.6-4.95) were associated with the highest risk of a return encounter to the ER (Figure 3).

\section{Discussion}

\section{Main findings}

Emergency rooms are over-burdened, with hallway medicine becoming the norm in many healthcare centers. Studies in the United States and Canada have reported a significant increase in the number of ER encounters over the last decade. ${ }^{9,10}$ Post-surgical ER encounters have been estimated to cost 800-1000 dollars per encounter. ${ }^{11}$ Identifying strategies to reduce unnecessary ER encounters is one way to reduce the burden on strained systems, this has become even more important during the COVID-19 pandemic as we aim to reduce unnecessary encounters. Critical to developing interventions to reduce unnecessary encounters is a clear understanding of rates and characteristics of patients presenting for care. This study demonstrates that emergency room encounters following outpatient urologic surgery are common. Patients receiving urological 
surgery contributed the highest volume (47.5\%) and second highest rate of return ER encounters $(16 \%)$ compared to general surgery, gynecologic surgery, and thoracic surgery.

\section{Interpretation and comparison to other studies}

There has been limited study into ER encounters following urologic surgery. Most studies have focused on return admissions following major oncologic procedures. ${ }^{12-14}$ Variable readmission rates between 3-20\% have been reported, with procedure type being the main factor that determines the readmission rate. ${ }^{12-14}$ While outcomes of major oncologic procedures are important, as these surgeries may be morbid, this study differed from most in the literature as it focused on high volume outpatient surgeries which contribute much greater patient volumes overall compared to large inpatient surgeries.

This study was able to differentiate patients seen in the ER and subsequently admitted to hospital compared to those seen in the ER and discharged home. The group of patients that were discharged from the ER, and did not require admission, is an important population to study as they represent encounters to the ER that could potentially be prevented by more rapid access to a urologist or general medical clinic. In our study, $\sim 94 \%$ of post urology surgery ER encounters (2520 encounters) did not require admission. Assessment of these patients revealed that the most common causes were directly associated with urologic care (urinary retention, hematuria, etc.). These results suggest that targeted improvement programs could benefit our patients. If these patients could be redirected from the ER to outpatient clinics, virtual encounters, or nursing care, patient care would be improved and healthcare costs would be reduced.

Risk factors for an ER encounter after surgery such as a higher ASA score have been consistently identified across several studies examining readmissions and ER encounters following outpatient surgery. ${ }^{5,11,12,14,15}$ Despite this, the specific surgical procedure performed appears to be the driving factor. In a single-center study assessing all outpatient surgeries, urologic surgeries were found to have a several fold higher rate of ER return compared to nonurologic procedures, and a rate of return ER encounter similar to previous studies at $5.8 \% .{ }^{15}$ In one study tracking outcomes following transurethral resection of the prostate (TURP), a reported 30-day return rate to the ER of $14.4 \%$ was observed. ${ }^{16}$ An analysis by the Canadian Institute for Health Information (CIHI) across Ontario, Alberta, and the Yukon identified that endoscopic interventions involving the upper urinary tract and TURP procedures had the highest rate of ER visits out of all surgical procedures, with post-surgery ER visits rates within 30 days of $15.2 \%$ and $11.7 \%$ respectively. ${ }^{17}$ Our results have further confirmed this, showing that similar endoscopic procedures such as TURBT remain a key risk factor for post-operative ER encounters, with a similar return ER encounter rate of $17 \%$. This suggests that any intervention to improve the rate of return to the ER should focus on high-risk procedures, rather than highrisk patients. 


\section{Strengths and limitations}

This study has several strengths compared to previous publications. First, it includes data collected over approximately ten years, indicating the trends are not transient. With the ability to track ER encounters, readmission rates, reasons surrounding the encounter, and procedurespecific details, it provides a comprehensive evaluation of patients presenting to the ER following urologic outpatient surgery compared to what is available in the literature.

This study is based on data from a tertiary referral center, it is possible hospitals with different case mixes would have different rates. It is possible the tertiary hospital setting creates a bias towards higher rates of return encounters due to higher surgical complexity and patient morbidity and may not be generalizable to centers with different procedure and patient demographics. Adjustment was not done for individual surgeon specific variance given the large number of surgeons performing several different procedures. Data from ER encounters at other emergency departments in the region were not captured, therefore encounter rates reported in this study likely underestimate the true rates. When comparing urologic procedures to the other surgical services studied there may be some inherent bias or error introduced by comparing different procedure types across different specialties. However, the goal of this study was to evaluate return visits to the ER after outpatient surgery, and in that respect all the specialties studied shared common ground in that only procedures deemed to be outpatient surgeries were included. As a result, different procedures may vary in associated morbidity, but all were deemed to not represent a barrier to safe same-day discharge from hospital. Finally, we are not able to directly determine if ER encounters were attributable to the surgery compared to another element of the patient's disease process, or for another medical condition.

\section{Conclusions and implications for practice and future research}

This study of a Canadian single-center experience shows that ER encounters following outpatient surgery are a common occurrence. In comparison to our surgical colleagues in general surgery, gynecology and thoracic surgery, urology has both a high volume of patients and a high rate of return encounters to the ER. Future study is needed to further evaluate the nature and severity of the causes of return to the emergency room, to allow further stratification of these issues prior to intervention design. Although not studied specifically in a urologic outpatient surgery cohort, enhanced recovery after surgery (ERAS) protocols for outpatient surgeries do appear to reduce ER visits in general surgery patients, and novel techniques such as these should be studied in the urologic realm. ${ }^{18,19}$ Surgical procedure appears to be a clear risk factor for return to the ER, and perhaps more involved follow up strategies for these procedures (early post-discharge telehealth call or office visit) may help to reduce subsequent emergency room use. With further analyses targeted strategies to prevent these ER encounters represent a significant opportunity where improvements to our patient's quality of life and reduced costs to our health system may be achieved. 


\section{References}

1. Kocher KE, Nallamothu BK, Birkmeyer JD, et al: Emergency Department Visits After Surgery Are Common For Medicare Patients, Suggesting Opportunities To Improve Care. Health Aff. (Millwood) 2013; 32: 1600-1607.

2. Hospital Readmissions Reduction Program (HRRP). Center for Medicare and Medicaid Services. https://www.cms.gov/Medicare/Medicare-Fee-for-ServicePayment/AcuteInpatientPPS/Readmissions-Reduction-Program, accessed April 9, 2020.

3. Ghosh S and Sallam S: Patient satisfaction and postoperative demands on hospital and community services after day surgery. BJS Br. J. Surg. 1994; 81: 1635-1638.

4. $\mathrm{Ag} \mathrm{P}$ and $\mathrm{Pu} \mathrm{R}$ : Patient satisfaction and postoperative demands on hospital and community services after day surgery. Br. J. Surg. 1995; 82: 714-715.

5. Mezei G and Chung F: Return Hospital Visits and Hospital Readmissions After Ambulatory Surgery. Ann. Surg. 1999; 230: 721.

6. Aylin P, Williams S, Jarman B, et al: Trends in day surgery rates. BMJ 2005; 331: 803.

7. Toftgaard C: World Wide Day Surgery Activity 2003 IAAS Survey of Ambulatory Surgery. Ambul. Surg. 2007: 8.

8. Cancer Quality Council of Ontario. https://www.csqi.on.ca/, accessed January 17, 2021.

9. Sun R, Karaca Z and Wong HS: Trends in Hospital Emergency Department Visits by Age and Payer, 2006-2015: Statistical Brief \#238. In: Healthcare Cost and Utilization Project (HCUP) Statistical Briefs. Rockville (MD): Agency for Healthcare Research and Quality (US) 2006. Available at: http://www.ncbi.nlm.nih.gov/books/NBK513766/, accessed April 20, 2020.

10. Emergency Department Time Spent by Patients in Ontario. Health Quality Ontario (HQO). https://www.hqontario.ca/System-Performance/Time-Spent-in-EmergencyDepartments, accessed April 20, 2020.

11. Coley KC, Williams BA, DaPos SV, et al: Retrospective evaluation of unanticipated admissions and readmissions after same day surgery and associated costs. J. Clin. Anesth. 2002; 14: 349-353.

12. Leow JJ, Gandaglia G, Sood A, et al: Readmissions after major urologic cancer surgery. Can. J. Urol. 2014; 21: 7537-7546.

13. Stone BV, Cohn MR, Donin NM, et al: Evaluation of Unplanned Hospital Readmissions After Major Urologic Inpatient Surgery in the Era of Accountable Care. Urology 2017; 109: 94-100.

14. Schmid M, Chiang HA, Sood A, et al: Causes of hospital readmissions after urologic cancer surgery. Urol. Oncol. Semin. Orig. Investig. 2016; 34: 236.e1-236.e11.

15. Twersky R, Fishman D and Homel P: What Happens After Discharge? Return Hospital Visits After Ambulatory Surgery. Anesth. Analg. 1997; 84: 319-324.

16. Palmisano F, Boeri L, Fontana M, et al: Incidence and predictors of readmission within 30 days of transurethral resection of the prostate: a single center European experience. Sci. Rep. 2018; 8: 1-7.

17. Canadian Institute for Health Information. Available at: https://www.cihi.ca/en, accessed December 18, 2020. 
18. Dumestre DO, Redwood J, Webb CE, et al: Enhanced Recovery After Surgery (ERAS) Protocol Enables Safe Same-Day Discharge After Alloplastic Breast Reconstruction. Plast. Surg. Oakv. Ont 2017; 25: 249-254.

19. Parrish AB, O’Neill SM, Crain SR, et al: An Enhanced Recovery After Surgery (ERAS) Protocol for Ambulatory Anorectal Surgery Reduced Postoperative Pain and Unplanned Returns to Care After Discharge. World J. Surg. 2018; 42: 1929-1938.

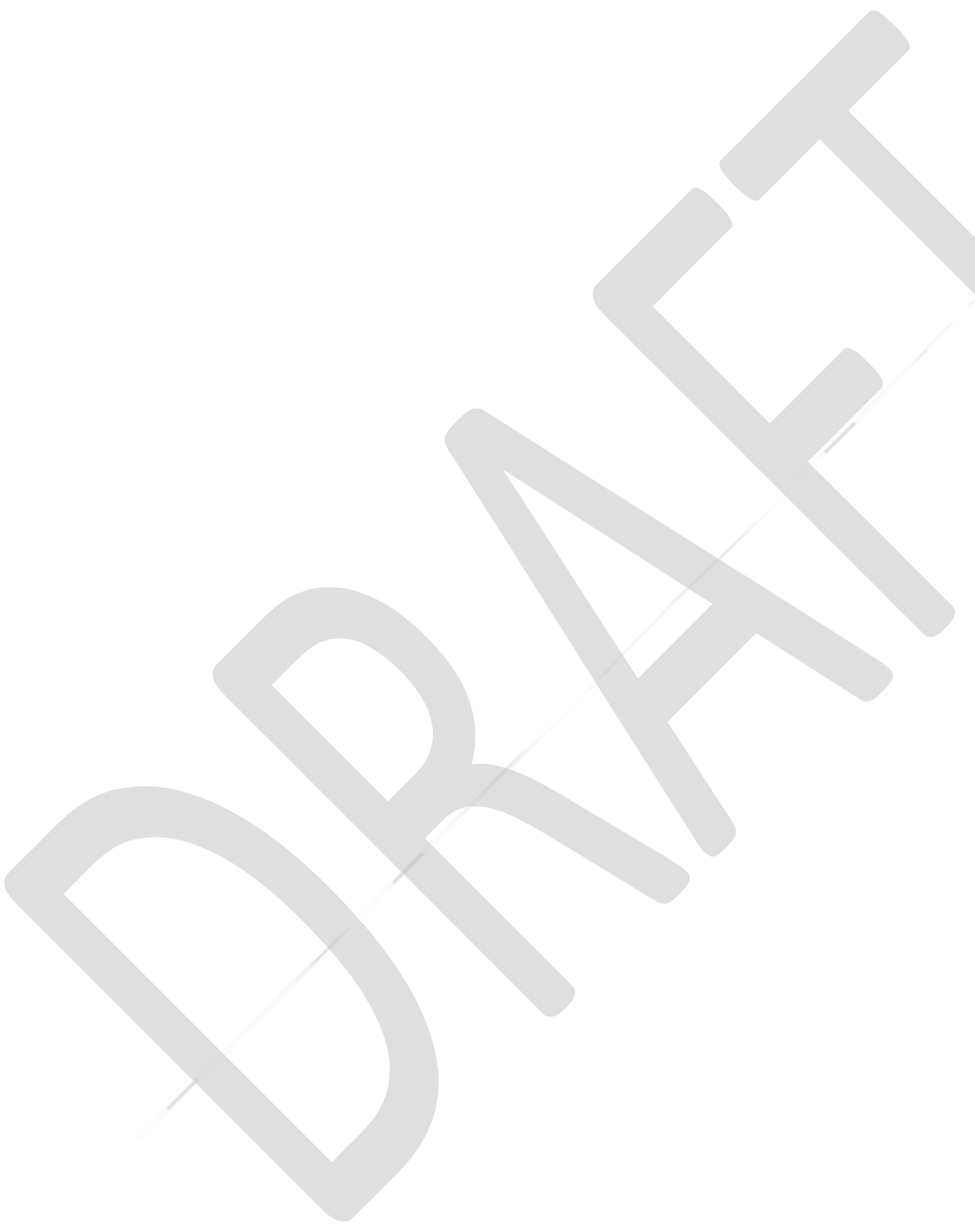




\section{Figures and Tables}

Fig. 1. Percent of emergency room encounters following outpatient surgery across four surgical services.

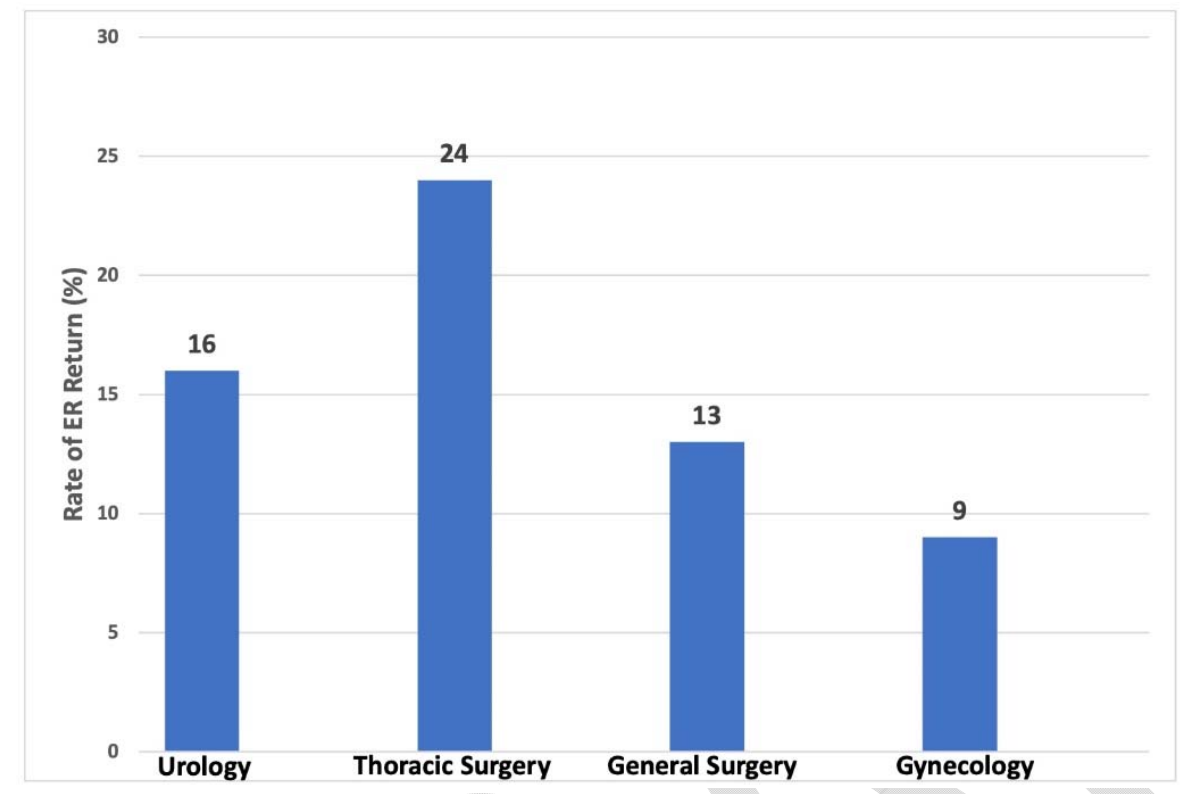

Fig. 2. Percent of emergency room encounters based on outpatient procedure. PD: peritoneal dialysis; PVP: photo vaporization of prostate; TURBT: transurethral resection of bladder tumor.

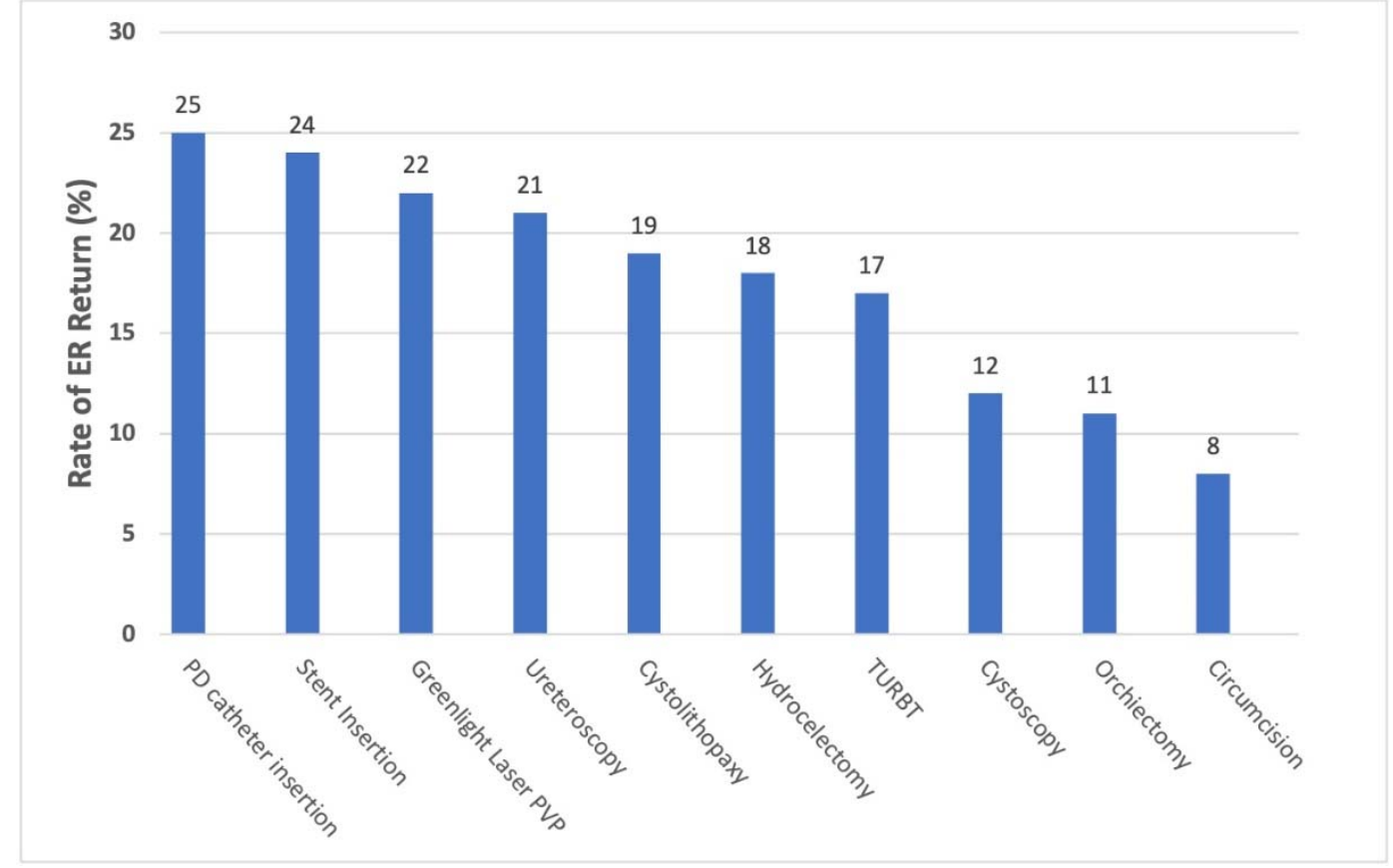


Fig. 3. Multivariate analysis of risk factors for return to emergency room after urologic outpatient surgery. ASA: American Society of Anesthesia; CI: confidence interval; PD: peritoneal dialysis; PVP: photo vaporization of prostate; RR: relative risk; TURBT: transurethral resection of bladder tumor. Reference procedure: circumcision.

Yes vs. Not married

Patient age increase by 5 year Surgery year increase by 1 year Yes vs. No primary care provider Sex female vs. male Yes vs. No presence of cancer

IV vs. I ASA score

IV vs. II ASA score

IV vs. III ASA score

PD catheter insertion vs. Reference procedure Stent Insertion vs. Reference procedure Greenlight Laser PVP vs. Reference procedure Ureteroscopy vs. Reference procedure Cystolithopaxy vs. Reference procedure Hydrocelectomy vs. Reference procedure TURBT vs. Reference procedure Cystoscopy vs. Reference procedure Orchiectomy vs. Reference procedure

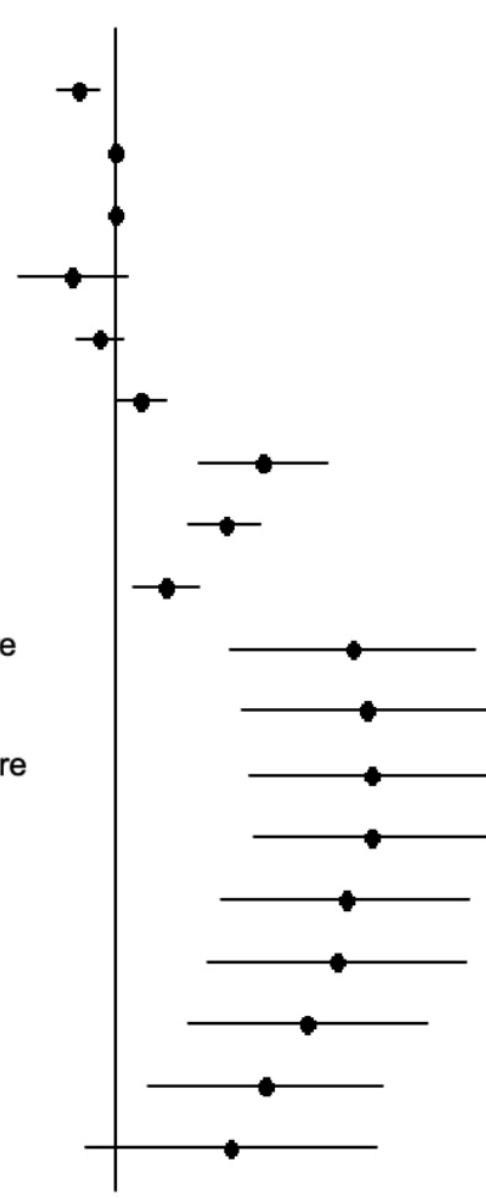

$\operatorname{RR}(95 \% \mathrm{Cl})$

$0.9(0.8,0.9)$

$1.0(1.0,1.0)$

$1.0(1.0,1.0)$

$0.8(0.6,1.1)$

$0.9(0.8,1.0)$

1.1(1.0,1.3)

2.0(1.5,2.6)

1.6(1.4,1.9)

1.3(1.1,1.5)

$2.9(1.7,5.1)$

$3.1(1.8,5.5)$

$3.2(1.8,5.6)$

$3.2(1.9,5.4)$

2.8(1.0.6,5)

$2.7(1.5,4.9)$

$2.4(1.4,4.1)$

$2.0(1.2,3.3)$

$1.7(0.9,3.3)$

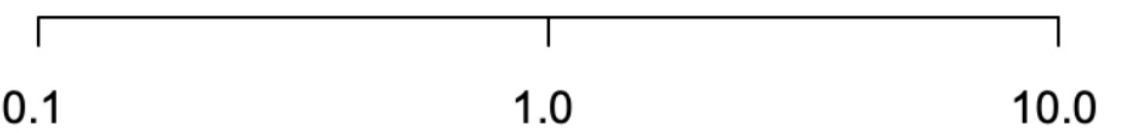




\begin{tabular}{|c|c|c|c|c|}
\hline & Urology & $\begin{array}{l}\text { Thoracic } \\
\text { surgery }\end{array}$ & $\begin{array}{l}\text { General } \\
\text { surgery }\end{array}$ & Gynecology \\
\hline Patient age, years (SD) & $63.3(16.4)$ & $64.6(12.4)$ & $52.9(15.5)$ & $49.8(14.2)$ \\
\hline $\begin{array}{l}\text { Number of procedures performed, } \\
\mathrm{n}(\% \text { of total procedures })\end{array}$ & $16552(43.1)$ & $1866(4.9)$ & $18676(48.7)$ & $1283(3.3)$ \\
\hline $\begin{array}{l}\text { Sex, n }(\%) \\
\text { Male } \\
\text { Female }\end{array}$ & $\begin{array}{c}12393(74.9) \\
4159(25.1)\end{array}$ & $\begin{array}{l}944(50.6) \\
922(49.4)\end{array}$ & $\begin{array}{c}7663(41.0) \\
11013(59.0)\end{array}$ & $\begin{array}{c}0 \\
1283(100)\end{array}$ \\
\hline $\begin{array}{l}\text { Number of ER encounters, } \mathrm{n}(\% \text { of } \\
\text { total encounters) }\end{array}$ & $2681(16.2)$ & $440(23.6)$ & 2404 (12.9) & $116(9.0)$ \\
\hline $\begin{array}{l}\text { Number of ER encounters, } \mathrm{n}(\% \text { of } \\
\text { total ER encounters) } \\
\quad 1 \\
2 \\
>2\end{array}$ & $\begin{array}{l}1805(67.3) \\
559(20.9) \\
317(11.8) \\
\end{array}$ & $\begin{array}{c}296(67.3) \\
98(22.3) \\
46(10.5) \\
\end{array}$ & $\begin{array}{c}1750(72.8) \\
459(19.1) \\
195(8.1) \\
\end{array}$ & $\begin{array}{r}92(79.3) \\
17(14.7) \\
8(6.9) \\
\end{array}$ \\
\hline $\begin{array}{l}\text { Days between surgery and ER } \\
\text { encounter, } \mathrm{n} \text { ( } \% \text { to ER encounters) } \\
\leq 14 \text { days } \\
15-30 \text { days } \\
>30 \text { days }\end{array}$ & $\begin{array}{l}1378(51.4) \\
416(15.5) \\
887(33.1)\end{array}$ & $\begin{array}{l}104(23.6) \\
100(22.7) \\
236(53.6)\end{array}$ & $\begin{array}{l}1302(54.2) \\
324(13.5) \\
778(32.4)\end{array}$ & $\begin{array}{l}44(37.9) \\
22(19.0) \\
50(43.1)\end{array}$ \\
\hline $\begin{array}{l}\text { Age of ER encounter (years), n (\%) } \\
\quad<40 \\
40-60 \\
>60\end{array}$ & $\begin{array}{c}269(10.1) \\
663(24.7) \\
1749(65.2)\end{array}$ & $\begin{array}{c}18(4.1) \\
125(28.4) \\
297(67.5)\end{array}$ & $\begin{array}{l}489(20.3) \\
1084(45.1) \\
831(34.6)\end{array}$ & $\begin{array}{l}34(29.3) \\
52(44.8) \\
30(25.9) \\
\end{array}$ \\
\hline
\end{tabular}

ER: emergency room; SD: standard deviation. 


\begin{tabular}{|l|}
\hline $\begin{array}{l}\text { Supplementary Table 1. Top urology surgical procedures by volume in } \\
\text { decreasing order }\end{array}$ \\
\hline Cystoscopy \\
\hline Transurethral resection of bladder tumor \\
\hline Cystolithopaxy \\
\hline Ureteroscopy \\
\hline Peritoneal dialysis catheter insertion \\
\hline Greenlight vaporization of prostate \\
\hline Hydrocelectomy \\
\hline Circumcision \\
\hline Ureteric stent insertion \\
\hline Orchiectomy \\
\hline Vasovasostomy \\
\hline Direct visual urethrotomy \\
\hline Suprapubic catheter insertion \\
\hline Spermatocelectomy \\
\hline Transurethral resection of bladder neck \\
\hline
\end{tabular}

Procedures included in analysis but not listed in supplemental table represent procedures performed $<1$ times per month over study period. 